\title{
Statistical compared to clinical significance and the risk of misattribution
}

\author{
To the Editor:
}

We read with interest the observational analysis by VozorIs et al. [1] of the putative association between the prescription of new antidepressants (selective serotonin reuptake inhibitors (SSRIs) or serotonin-noradrenaline reuptake inhibitors (SNRIs)), and hospitalisation and mortality in people with a diagnosis of chronic obstructive pulmonary disease (COPD). In this nonrandomised analysis of administrative health data, people who got a new prescription of these antidepressants faced a worse prognosis including a $26 \%$ increase in pneumonia-related mortality and a $20 \%$ higher risk of death from all causes [1]. This analysis used similar methodology as previous papers on benzodiazepines and opioids and shares the same important shortcomings $[2,3]$.

The associations between antidepressant prescription and increased hospitalisation and mortality likely reflect the underlying illness(es) and other unmeasured factors rather than an actual drug effect; the authors acknowledge the general inability of observational studies to establish causative relationships. This analysis, therefore, does not support the authors' conclusions that these findings "should prompt prescribers to consider the potential for increased respiratory-related morbidity and mortality in SSRI/ SNRI prescribing decision making" [4].

People who have a clinical encounter which results in a prescription for antidepressants are likely to differ from those who do not in important ways. The severity of underlying illness, in this case both of COPD or anxiety/depression, and the indication for the drug treatment are recognised as key confounders [5], neither of which can be controlled for in this dataset. In addition, data were unavailable on other important factors such as smoking exposure, lung function, alcohol consumption, socioeconomics, symptoms, dietary status and behaviours including fitness and activity. We acknowledge that the authors did what can be done to control for the limited factors available, but excluding people with a reimbursed palliative care consultation in a health system where only $5 \%$ of all decedents from COPD have a palliative care consultation [6] is clearly insufficient. The risk of death or hospitalisation is not equal for all people included in this cohort study. The findings of the study, therefore, reflect an association between expected deaths and symptom interventions offered to people with symptomatic life-limiting disease. In light of this, the moderately higher rates of hospitalisation and death in people with treatment is no surprise.

That the findings are related to confounding is supported by the fact that the increased risks disappeared when analysed only in people with diagnosed psychiatric illness. The reason for this is more likely better adjustment for underlying illness (confounding) than a biological rationale. There was also no doseresponse relationship. Of note, the large number of participants in the study increases precision but does not affect confounding, risking yielding a more precise estimate of a spurious association [7].

We are not suggesting that observational studies of potential drug effects should be avoided. However, crucial factors must be considered in the design and interpretation of these studies such as the pre-likelihood of a true effect (in line with a Baysian approach), the size of the effects, and the ability of the study to control for key confounders such as severity of the illness(es) related to the treatment and outcomes (both benefits and harms). The risk of confounding in observational studies is high when the treatment is strongly related to underlying illnesses which, in turn, have strong relationships with the studied outcomes. Using a similar design as in the present study, one may find substantial negative associations between treatment of any serious and distressing symptom and clinical outcomes, e.g. for bronchodilators for airflow limitation, for starting pulmonary rehabilitation, for use of mobility aids and

@ERSpublications

Observational analyses of putative drug effects, including a recent on antidepressants and increased mortality in COPD, may be biased, should be interpreted sceptically and often need to be confirmed by randomised data to change practice. http://ow.ly/oh5030m9Q9I

Cite this article as: Ekström M, Johnson MJ, Currow DC. Statistical compared to clinical significance and the risk of misattribution. Eur Respir J 2018; 52: 1801723 [https://doi.org/10.1183/13993003.01723-2018]. 
even for smoking cessation. Confounding is likely for all palliative interventions given to relieve symptoms in the setting of advancing disease.

We agree with the authors' statement that "absolute adverse event rates of our positive outcomes are relatively small, and therefore may not be clinically significant or they may not have remained positive had additional covariates been controlled for." The clinician (as well as other people) should remain sceptical, interpret the findings in the light of pre-likelihood of true effect and the risk of residual confounding, and await further evaluation of net-benefit using randomised data where possible before changing clinical practice.

Magnus Ekström ${ }^{1,2}$, Miriam J. Johnson ${ }^{3}$ and David C. Currow ${ }^{2,3}$

${ }^{1}$ Dept of Clinical Sciences, Division of Respiratory Medicine and Allergology, Lund University, Lund, Sweden. ${ }^{2}$ IMPACCT, Faculty of Health, University of Technology Sydney, Ultimo, Australia. ${ }^{3}$ Wolfson Palliative Care Research Centre, Hull York Medical School, University of Hull, Hull, UK.

Correspondence: Magnus Ekström, Dept of Medicine, Blekinge Hospital, SE-17385 Karlskrona, Sweden.

E-mail: pmekstrom@gmail.com

Received: Sept 102018 | Accepted: Sept 112018

Conflict of interest: M. Ekström has nothing to disclose. M.J. Johnson reports institutional fees for clinical advisor role from Mayne Pharma, during the conduct of the study. D.C. Currow reports an unrestricted research grant from Mundipharma, is an unpaid member of an advisory board for Helsinn Pharmaceuticals, and has consulted Specialist Therapeutics and Mayne Pharma and received intellectual property payments from Mayne Pharma.

\section{References}

1 Vozoris NT, Wang X, Austin PC, et al. Serotonergic antidepressant use and morbidity and mortality among older adults with COPD. Eur Respir J 2018; 52: 1800475.

2 Vozoris NT, Fischer HD, Wang X, et al. Benzodiazepine drug use and adverse respiratory outcomes among older adults with COPD. Eur Respir J 2014; 44: 332-340.

3 Vozoris NT, Wang X, Fischer HD, et al. Incident opioid drug use and adverse respiratory outcomes among older adults with COPD. Eur Respir J 2016; 8: 683-693.

4 Agoritsas T, Merglen A, Shah ND, et al. Adjusted analyses in studies addressing therapy and harm. Users' guides to the medical literature. JAMA 2017; 317: 748-759.

5 Norgaard M, Ehrenstein V, Vandenbroucke JP. Confounding in observational studies based on large health care databases: problems and potential solutions - a primer for the clinician. Clin Epidemiol 2017; 9: 185-193.

6 Gershon AS, Maclagan LC, Luo J, et al. End of life strategies among patients with advanced chronic obstructive pulmonary disease (COPD). Am J Respir Crit Care Med 2018; in press [https://doi.org/10.1164/rccm.201803-0592OC].

7 Kaplan RM, Chambers DA, Glasgow RE. Big data and large sample size: a cautionary note on the potential for bias. Clin Transl Sci 2014; 7: 342-346.

Copyright @ERS 2018

\section{From the authors:}

We thank M. Ekström and colleagues for their interest in our study [1] and for their comments. The central concepts that M. Ekström and colleagues express in their correspondence are that our findings of increased respiratory-related morbidity and mortality in association with new serotonergic antidepressant use among older adults with chronic obstructive pulmonary disease (COPD) "likely reflect the underlying illness(es) and other unmeasured factors rather than an actual drug effect".

Regarding the possibility of underlying COPD severity or the presence of psychiatric disease or other comorbidity accounting for the increased morbidity and mortality observed among new users of serotonergic antidepressants, it is important for M. Ekström and colleagues to recognise that we used propensity score matching methods in our study, which resulted in the balancing of multiple markers of COPD severity (including the frequency and recency of prior COPD exacerbation, duration of COPD and receipt of COPD medications) between treated and control individuals. Furthermore, balance was induced

@ERSpublications

Propensity score matching and stratification are methods that help minimise the risk of confounding and misattribution in observational studies http://ow.ly/DY0n30mrtQF

Cite this article as: Vozoris NT, Austin PC, Rochon PA. Statistical compared to clinical significance and the risk of misattribution. Eur Respir J 2018; 52: 1801868 [https://doi.org/10.1183/13993003.01868-2018]. 
between treated and control individuals on the presence of psychotic and non-psychotic psychiatric disease and on 11 additional comorbidity categories (in addition to the Johns Hopkins Adjusted Clinical Group, which is a validated measure of patient morbidity burden). Furthermore, we undertook several sensitivity analyses, whose purpose was to help further minimise the possibility of underlying illness severity influencing findings. We evaluated our outcomes stratifying by COPD exacerbation frequency history, which is known to be the strongest predictor of COPD morbidity [2]. We found significantly elevated rates of all-cause mortality among new users of serotonergic antidepressants, who had no previous COPD exacerbation, which would be the healthiest COPD subgroup and least likely to be influenced by confounding by indication. We also evaluated our outcomes in a sensitivity analysis where new tricyclic antidepressant users formed the control group (see the online supplementary material for our article [1]), because serotonergic antidepressants and tricyclic antidepressants have similar prescribing indications. Compared to new tricyclic antidepressant users, we observed significantly increased rates of hospital admission for COPD or pneumonia, COPD or pneumonia-related mortality and all-cause mortality among new serotonergic antidepressant users. In contrast to what M. Ekström and colleagues have written, the fact that increased rates of respiratory-related morbidity and mortality were observed among the subgroup of new serotonergic antidepressant users without pre-existing psychiatric disease (which is likely to be a healthier subgroup of individuals, compared to those with established psychiatric disease) again minimises the chances that our findings are influenced by confounding by indication. Finally, the fact that we used new drug receipt (but not of a serotonergic antidepressant) to define inclusion into the control group also helped serve to decrease the likelihood of underlying unstable health potentially explaining any differences observed between the exposed and control groups.

Regarding unmeasured factors not included in our propensity score model influencing our results, we acknowledged this possibility in our article [1]. However, for our findings to be "likely" to be explained by this possibility, as M. Ekström and colleagues have written, is simply speculation on their part. M. Ekström and colleagues offer no evidence to support the premise that, had additional covariates been adjusted for in our analysis, this would have been "likely" to have rendered our positive findings nonsignificant. It is also not clear how some of the unmeasured factors they listed, like alcohol consumption and dietary status, if indeed different between the exposed and control groups, would have possibly contributed to increased COPD-related morbidity and mortality. M. Ekström and colleagues also incorrectly list socioeconomics and smoking exposure as variables uncontrolled for in our analysis. In our propensity score model, several markers of socioeconomic status were included, as was receipt of smoking cessation pharmacotherapy within the previous year (which is a proxy measure for recent cigarette smoke exposure).

M. Ekström and colleagues write that using a similar observational study design to that which we have used, one might naturally find spurious, negative associations between established treatments for COPD (e.g. inhalers) and COPD outcomes, since receipt of treatment is related to underlying illness and underlying illness influences outcomes. However, this assertion is contradicted by a number of published observational studies evaluating the real-world effectiveness of combination versus single-agent inhaler therapy among individuals with COPD, where combination inhaler therapy (which may be more likely to be given to individuals with more symptomatic or unstable COPD, compared with a single-agent inhaler) was not found to be associated with increased rates of negative respiratory outcomes compared with single-agent inhaler therapy [3-5].

We agree with the concluding comment of M. Ekström and colleagues that further research is warranted (both in the form of randomised, placebo-controlled clinical trials and real-world observational studies) to more fully understand the potential benefits and harms of serotonergic antidepressants in the COPD population. It worth noting, however, that randomised clinical trials to date have failed to demonstrate that serotonergic antidepressant drugs are beneficial for either depression and anxiety [6-8], or refractory dyspnoea [8-10], among individuals with COPD.

Nicholas T. Vozoris ${ }^{1,2,3,4}$, Peter C. Austin ${ }^{4,5}$ and Paula A. Rochon ${ }^{3,4,5,6}$

${ }^{1}$ Division of Respirology, Dept of Medicine, St Michael's Hospital, Toronto, ON, Canada. ${ }^{2}$ Keenan Research Centre in the Li Ka Shing Knowledge Institute, St Michael's Hospital, Toronto, ON, Canada. ${ }^{3}$ Dept of Medicine, University of Toronto, Toronto, ON, Canada. ${ }^{4}$ Institute for Clinical Evaluative Sciences, Toronto, ON, Canada. ${ }^{5}$ Institute of Health Policy, Management, and Evaluation, University of Toronto, Toronto, ON, Canada. ${ }^{6}$ Women's College Research Institute, Women's College Hospital, Toronto, ON, Canada.

Correspondence: Nicholas T. Vozoris, Division of Respirology, Dept of Medicine, St Michael's Hospital, 30 Bond Street, Toronto, ON, M5B 1W8, Canada. E-mail: nick.vozoris@utoronto.ca

Received: Oct 022018 | Accepted: Oct 042018

Conflict of interest: None declared. 


\section{References}

1 Vozoris NT, Wang X, Austin PC, et al. Serotonergic antidepressant use and morbidity and mortality among older adults with COPD. Eur Respir J 2018; 52: 1800475

2 Hurst JR, Vestbo J, Anzueto A, et al. Susceptibility to exacerbation in chronic obstructive pulmonary disease. N Engl J Med 2010; 363: 1128-1138.

3 Gershon AS, Campitelli MA, Croxford R, et al. Combination long-acting $\beta$-agonists and inhaled corticosteroids compared with long-acting $\beta$-agonists alone in older adults with chronic obstructive pulmonary disease. JAMA 2014; 312: 1114-1121.

4 Trudo F, Kern DM, Davis JR, et al. Comparative effectiveness of budesonide/formoterol combination and tiotropium bromide among COPD patients new to these controller treatments. Int J Chron Obstruct Pulmon Dis 2015; 10: 2055-2066.

5 Cho KH, Kim YS, Linton JA, et al. Effects of inhaled corticosteroids/long-acting agonists in a single inhaler versus inhaled corticosteroids alone on all-cause mortality, pneumonia, and fracture in chronic obstructive pulmonary disease: a nationwide cohort study 2002-2013. Respir Med 2017; 130: 75-84

6 Usmani ZA, Carson KV, Cheng JN, et al. Pharmacological interventions for the treatment of anxiety disorders in chronic obstructive pulmonary disease. Cochrane Database Syst Rev 2011; 11: CD008483.

7 Evans M, Hammond M, Wilson K, et al. Placebo-controlled treatment trial of depression in elderly physically ill patients. Int J Geriatr Psychiatry 1997; 12: 817-824.

8 Lacasse Y, Beaudoin L, Rousseau L, et al. Randomized trial of paroxetine in end-stage COPD. Monaldi Arch Chest Dis 2004; 61: 140-147.

9 Eiser N, Harte R, Spiros K, et al. Effect of treating depression on quality-of-life and exercise tolerance in severe COPD. COPD 2005; 2: 233-241.

10 Yohannes AM, Connolly MJ, Baldwin RC. A feasibility study of antidepressant drug therapy in depressed elderly patients with chronic obstructive pulmonary disease. Int J Geriatr Psychiatry 2001; 16: 451-454. 